\title{
Generalized Interface Polarity Scale Based on Second Harmonic Spectroscopy
}

\author{
Hongfei Wang, ${ }^{\dagger}$ E. Borguet, ${ }^{\star}$ and K. B. Eisenthal* \\ Department of Chemistry, Columbia University, 3000 Broadway, New York, New York 10027 \\ Received: January 6, 1998; In Final Form: April 20, 1998
}

\begin{abstract}
Quantitative polarity scales have been of great value in advancing our understanding of chemical and physical processes in bulk solvents. However, an understanding of the polarity at liquid interfaces has been more elusive and no scale of interface polarity currently exists. Following our demonstration that second harmonic spectroscopy can be used to measure interface polarity, we have determined the polarity of several liquid/ liquid and vapor/liquid interfaces. The polarities of the water/1,2-dichloroethane and water/chlorobenzene interfaces have been investigated using the polarity indicator molecule $N, N$-diethyl- $p$-nitroaniline (DEPNA). The betaine dye 4-(2,4,6-triphenylpyridinium)-2,6-diphenylphenoxide, $\left(E_{\mathrm{T}}(30)\right)$, was used to probe the polarity of the air/water interface. The intramolecular charge transfer (CT) absorption band positions of both DEPNA and $E_{\mathrm{T}}(30)$ are measured and used to define the interface solvent polarity. An important finding is that the polarity of the liquid interfaces is simply related to the polarity of the bulk phases. The interface polarity is found to be equal to the arithmetic average of the polarity of the adjoining bulk phases. This surprisingly simple result suggests the possible dominance of the long-range solute-solvent interactions, not the local interface interactions, in determining the difference in the excited- and ground-state solvation energies of the interface adsorbed molecules.
\end{abstract}

\section{Introduction}

Solvent polarity is a catch-all phrase that is used to describe a range of intermolecular interactions between solute molecules and the solvent in which they are immersed. The organization of solvent molecules about a solute molecule alters the electronic and vibrational energies of the solute's ground and excited states, and more generally the potential surfaces, intersections, and barriers that separate different molecular states and structures. As a consequence of these perturbations, the nonradiative energy relaxation processes in a solute molecule can be strongly affected by the solvent polarity. Thus, the efficiency and dynamics of competing chemical, as well as physical, pathways are often sensitive to the solvent polarity. This is especially the case when the chemical or physical process involves a change in charge distribution along the reaction or molecular motion coordinates. To discuss the role of solvent polarity in a quantitative manner, solvent polarity scales have been devised. These scales, which are based on solvent-dependent molecular properties, ${ }^{1-4}$ have enabled chemical and physical behavior in liquids to be rationalized and predictions made in a simple and intuitive manner. ${ }^{1-4}$ It has been shown, for example, that in bulk solutions, the solvent dependence of molecular isomerization rates of the cyanine dye DODCI is explained using solvent polarity scales. ${ }^{5 a}$ Other examples include the rate of intersystem crossing of aromatic carbenes ${ }^{5 b}$ and the twisted intramolecular chargetransfer (TICT) dynamics in polar solvents. ${ }^{6}$ In a similar way a knowledge of interface polarity has the potential of providing understanding of a wide range of interfacial phenomena. However, unlike the bulk liquid, no scale of interface polarity currently exists.

\footnotetext{
$\dagger$ Present address: Department of Chemistry, University of Pennsylvania Philadelphia, PA 19104.

$\doteqdot$ Permanent address: Department of Chemistry, University of Pittsburgh, Pittsburgh, PA 15260

* To whom correspondence should be addressed.
}

It is already well documented that molecular behavior at interfaces is different from that in bulk media., ${ }^{7,8}$ This is exemplified by a number of different observations, such as differences between $\mathrm{p} K_{\mathrm{a}}$ 's at interfaces and in bulk liquids. The observation that the shift in acid-base equilibria favors the neutral form at the air-water interface has been rationalized by assuming that the interface is a less polar region than the bulk aqueous solution. Charged species are less completely solvated at the interface and experience image repulsions at the air/water interface, which raises the free energy of the charged form more than the neutral form. As a consequence, the neutral, less polar form is favored at the interface compared to the bulk. However reasonable this argument may be, it has not been quantitatively tested; the polarity of the air/water interface had not been determined, prior to our recent report, ${ }^{9}$ and is not listed on any polarity scale. Similarly, the quantitative description of ionic adsorption at liquid interfaces relies on estimated values of interfacial polarity that are presently unknown. Clearly, a knowledge of interface polarity and a means of predicting interface polarity would be most useful. This is precisely what the present paper proposes.

Earlier investigations of polarity in the interface region have focused on systems such as water-in-oil microemulsions, ${ }^{10}$ oilin-water microemulsions, ${ }^{11}$ ionic and nonionic micelles, ${ }^{12,13}$ and alumina ${ }^{14}$ and silica ${ }^{15}$ interfaces. A polarity indicator molecule, 4-(2,4,6-triphenylpyridinium)-2,6-diphenylphenoxide $\left(E_{\mathrm{T}}(30)\right)$, has also been investigated at free oil/water interfaces by visible attenuated total internal reflectance absorption spectroscopy. ${ }^{16}$ Unlike second harmonic generation (SHG) and sum frequency generation (SFG), the techniques employed in these aforementioned studies are not necessarily interface-specific. It is not clear, therefore, whether the bulk media polarity affected the results obtained. It would be useful to compare SHG findings on interface polarity with the results of these other methods. 
CHART 1: $\quad N, N$-Diethyl-p-nitroaniline (DEPNA)<smiles>CC[NH+](CC)c1ccc([N+](=O)[O-])cc1</smiles>

CHART 2: 4-(2,4,6-Triphenylpyridinium)-2,6diphenylphenoxide $\left(E_{\mathrm{T}}(30)\right)$

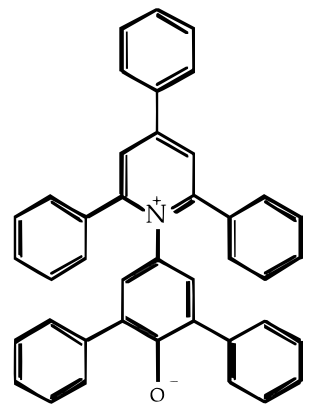

Recently, we reported the first measurement of the polarity of the air/water interface. ${ }^{9}$ This was achieved using second harmonic generation to measure the charge-transfer transition energy of the polarity-sensitive molecule $N, N$-diethyl- $p$-nitroaniline, DEPNA ${ }^{7,8,17,18}$ (Chart 1). DEPNA is one of a family of solvatochromic nitroaromatic indicators. ${ }^{3,19}$ Their solvatochromic properties are associated with a $\pi-\pi^{*}$ charge transfer (CT) transition involving charge density at the nitro and anilino substituents. On the basis of these nitroaromatic compounds, a series of correlated $\pi^{*}$ scales of solvent polarity have been constructed. The correlated $\pi^{*}$ value for a given solvent is given by ${ }^{19}$

$$
\pi^{*}=\frac{v_{\max }-27.52}{-3.182}
$$

where $v_{\max }$ is the absorption band peak of DEPNA in that solvent, in units of $10^{3} \mathrm{~cm}^{-1}$. For DEPNA the $\pi-\pi^{*}$ band peaks at $429 \mathrm{~nm}$ in bulk water $\left(\pi^{*}=1.32\right)$, which is the most polar solvent on the scale. The transition peaks at $359 \mathrm{~nm}$ in bulk $n$-hexane $\left(\pi^{*}=-0.11\right)$, which is the most nonpolar solvent on the scale. The principal finding of the SHG study was that for the air/water interface the charge-transfer peak band maximum occurs at $373 \mathrm{~nm}\left(\pi^{*}=0.22\right)$. This indicates that the polarity of the air/water interface is much less than that of bulk water and is close to that of nonpolar bulk solvents such as carbon tetrachloride $\left(\pi^{*}=0.26\right)$ and butyl ether $\left(\pi^{*}=0.21\right)$.

To develop a general interface polarity scale, it is necessary to establish that the scale is not dependent on the polarity probe molecule used in the study or that any differences can be understood in ways analogous to bulk polarity scales that use different polarity probe molecules. For this reason, we extended our polarity investigations to include a different polarity indicator molecule, a betaine dye 4-(2,4,6-triphenylpyridinium)-2,6diphenylphenoxide, $E_{\mathrm{T}}(30)$ (Chart 2). The bulk polarity scale based on its solvatochromic behavior is known as the $E_{\mathrm{T}}(30)$ scale. It is probably the most comprehensive and simply defined of the more than 30 polarity scales. ${ }^{1,20}$ Its intramolecular CT absorption band $\left(\pi-\pi^{*}\right)$ shows a large solvent shift; the peak moves from $453 \mathrm{~nm}$ in bulk water to $926 \mathrm{~nm}$ in bulk $n$-hexane. The $E_{\mathrm{T}}(30)$ value for a solvent is the electronic transition energy of the molecule in that solvent, in units of $\mathrm{kcal} / \mathrm{mol}$. It is given by

$$
E_{\mathrm{T}}(30)=\frac{28591}{\lambda_{\max }(\mathrm{nm})}
$$

$\lambda_{\text {max }}$ is the peak wavelength of its longest intramolecular chargetransfer absorption band $\left(\pi-\pi^{*}\right)$. A normalized value $E_{\mathrm{T}}^{\mathrm{N}}$ is often used and defined as

$$
E_{\mathrm{T}}^{\mathrm{N}}=\frac{E_{\mathrm{T}}(\text { solvent })-E_{\mathrm{T}}(\mathrm{TMS})}{E_{\mathrm{T}}(\text { water })-E_{\mathrm{T}}(\mathrm{TMS})}=\frac{E_{\mathrm{T}}(\text { solvent })-30.7}{32.4}
$$

using water and tetramethylsilane (TMS) as extreme polar and nonpolar reference solvents, respectively. Water has an $E_{\mathrm{T}}^{\mathrm{N}}$ value of 1.00 and $n$-hexane a value of $0.009 .{ }^{20}$

Aside from the widespread use of $E_{\mathrm{T}}(30)$ as a bulk polarity indicator, we are motivated to use it because the solvatochromic mechanisms of DEPNA and $E_{\mathrm{T}}(30)$ are different. ${ }^{24,25}$ The positive solvatochromism of DEPNA (spectral red shift as the solvent polarity increases) reflects the fact that DEPNA's excited state is more polar than its ground state. The negative solvatochromism of $E_{\mathrm{T}}(30)$ indicates that the opposite behavior is operative in this molecule. It is well-known that even though there is good qualitative correlation between the DEPNA's $\pi^{*}$ and $E_{\mathrm{T}}(30)$ polarity scales for bulk solvents, there is generally no one-to-one correspondence between the $\pi^{*}$ values and $E_{\mathrm{T}^{-}}$ (30) values for many solvents. These differences have been extensively discussed in the literature. ${ }^{1-4,24,25}$ However, the fact that a similar polarity for the air/water interface is obtained for distinct molecules, whose ground and excited states respond differently to solvent polarity, strongly supports the contention that the measured value is a good representation of the air/water interface polarity.

The present work further supports the generality of an interface polarity scale based on SHG measurements. Of particular importance is the finding that a simple analytical expression can be used to predict the interface polarity based on the known bulk polarities of the two constituent bulk phases.

\section{Experimental Section}

$E_{\mathrm{T}}(30)$ was obtained from two commercial sources: Aldrich and Fluka. Both gave the same UV-vis absorption spectra and experimental results. The $\mathrm{p} K_{\mathrm{a}}$ of $E_{\mathrm{T}}(30)$ is about $9 .{ }^{11}$ Bulk aqueous solution $\mathrm{pH}$ was kept at 12 using $\mathrm{NaOH}$ solutions to maintain $E_{\mathrm{T}}(30)$ in its neutral, polarity indicator form. The $E_{\mathrm{T}}-$ (30) concentration used in the SHG experiments was $5 \mu \mathrm{M}$. DEPNA was initially synthesized and purified by a standard literature procedure. ${ }^{21}$ Later it was purchased (Frinton Laboratories, $\mathrm{NJ}$ ) and used without further purification. Both samples gave the same experimental results. 1,2-Dichloroethane (DCE) and chlorobenzene (CB) were supplied by ACROS and Aldrich, respectively, and were of the highest spectroscopic grade available.

The SHG spectroscopy setup has been described previously. ${ }^{9}$ Briefly, a tunable, femtosecond Ti:sapphire oscillator is focused at the sample interface and second harmonic $(\mathrm{SH})$ photons are detected in the reflected direction using single-photon counting while the fundamental and background were blocked with filters and monochromator. Experiments involving liquid/liquid interfaces were performed in a cylindrical optical quartz cell (path length $=5 \mathrm{~cm}$, diameter $=5 \mathrm{~cm}$, from NSG Precision Cells, 


\section{CHART 3}

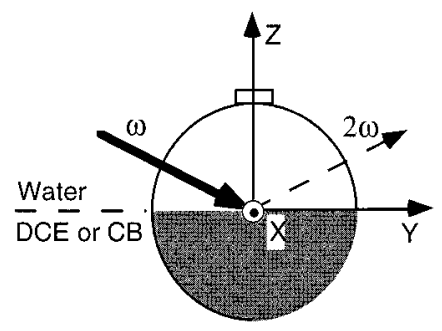

Inc.) (Chart 3). A large cell provides a relatively flat interface at the center of the cell, which facilitates the optical alignment. The high solubility of DEPNA in the organic phases $(>20 \mathrm{mM})$ and much smaller solubility in water $(<100 \mu \mathrm{M})$ ensure negligible partition of DEPNA into the water phase. This translates into a negligible optical absorption of the fundamental, since the SH radiated from the interface passes through the upper, less optically dense aqueous phase. Doubly distilled water was used to prepare all aqueous solutions. Experiments were performed at $22 \pm 0.5^{\circ} \mathrm{C}$.

The SH signals $\left(I^{(2)}\right)$ from the interfaces of the indicator solutions were normalized to the $\mathrm{SH}$ signal of the relevant neat liquid interface, air/water, water/DCE, or water/CB, which serves as wavelength-independent references. ${ }^{9}$ The normalization for each element of the susceptibility directly yields the square of $\chi^{(2) I N D I C A T O R} / \chi^{(2) \text { solvent }}$, where $\chi^{(2) I N D I C A T O R}$ is the susceptibility of the interface containing the indicator and $\chi^{(2) \text { solvent }}$ is the susceptibility with no indicator present. The normalized SH signal is noted as $I_{I J K}^{(2) I N D I C A T O R}$ in the text, with $I J K$ as $Z X X$ or $X Z X$, where $\mathrm{Z}$ is the lab axis normal to the interface and $X$ is the lab axis in the plane of the interface.

\section{Results and Discussion}

(a) $\boldsymbol{E}_{\mathrm{T}}(\mathbf{3 0})$ at the Air/Water Interface. The surface tension of the $E_{\mathrm{T}}(30)$ solution is the same as that of the air/neat water interface within our measurement accuracy $( \pm 0.1 \mathrm{dyn} / \mathrm{cm})$, indicating a low surface density of $E_{\mathrm{T}}(30)$ molecules ( $>4000$ $\AA^{2} /$ molecule).

The $\mathrm{SH}$ spectrum of $E_{\mathrm{T}}(30)$ at the air/water interface, which is one-photon resonant in the region of the fundamental laser wavelength, is shown in Figure 1. As discussed previously, the peak in the SH spectrum does not necessarily coincide with the peak of the $\pi-\pi^{*} \mathrm{CT}$ transition peak because of interference effects. ${ }^{9,22}$ The peak of the CT band of the interface species can be determined when the interference between the resonant and nonresonant terms is accounted for using an expression such as eq 4 , used previously. ${ }^{9}$

$$
I_{I J K}^{(2) E_{\mathrm{T}}(30)}=\left|1+\frac{a^{\prime}}{\left(\frac{1}{\lambda_{\max }}-\frac{1}{\lambda}+\mathrm{i} \Gamma^{\prime}\right)}+b\right|^{2}
$$

where $a^{\prime}, b, \Gamma^{\prime}$, and $\lambda_{\max }$ are the amplitudes of $\chi_{I J K, \text { Resonant }}^{(2) E_{\mathrm{T}}(30)}$ and $\chi_{I J K, \text { Nonresonant }}^{(2) E_{\mathrm{T}}(30)}$, the line width, and the transition peak wavelength, respectively. $\lambda$ is the fundamental (laser) wavelength. The fits of $I_{X Z X}^{(2) \mathrm{E}_{\mathrm{T}}(30)}$ and $I_{Z X X}^{(2) \mathrm{E}_{\mathrm{T}}(30)}$ to eq 4 are shown in Figure 1, yielding $\lambda_{\max }$ of $920 \pm 7 \mathrm{~nm}$ for the $X Z X$ element and $919 \pm$ $10 \mathrm{~nm}$ for the $Z X X$ element. The SH spectra are not complete, owing to the limits of tunability of the laser with the existing optics set. However, the data are sufficient to identify the peak position. Allowing for this, we place an upper limit on the location of the peak position of $\chi_{I J K, \text { Resonant }}^{(2) E_{\mathrm{T}}(30)}$ of $20 \mathrm{~nm}$, i.e., 920 $\pm 20 \mathrm{~nm}$.

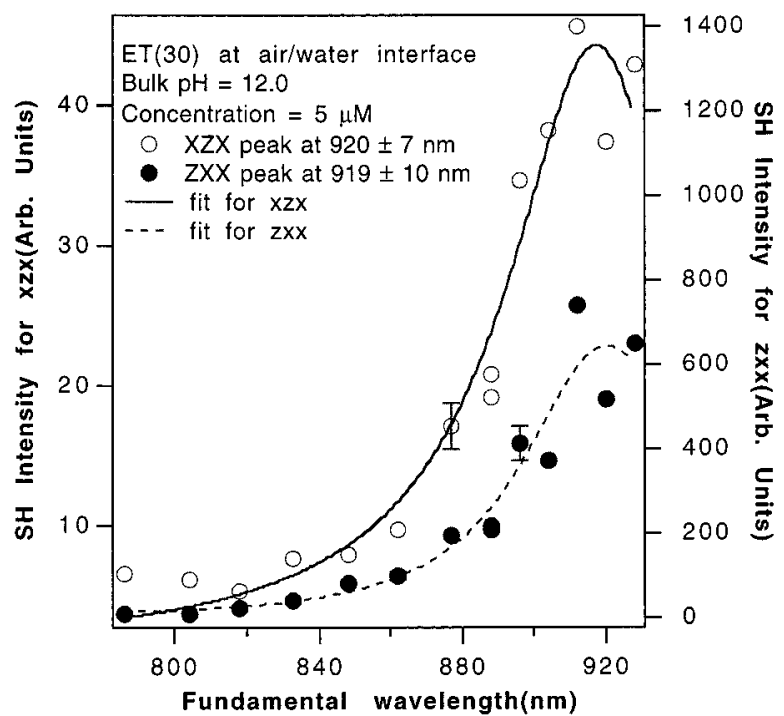

Figure 1. SH spectrum of $E_{\mathrm{T}}(30)$ at the air/water interface. The solid and dotted lines are fit to eq 4, yielding $\lambda_{\max }$ of $920 \pm 7 \mathrm{~nm}$ for the $X Z X$ element and $919 \pm 10 \mathrm{~nm}$ for the $Z X X$ element.

The position of the $\mathrm{SH}$ peak indicates that the polarity of the air/water interface on the $E_{\mathrm{T}}(30)$ scale is in the range of the nonpolar solvents $n$-heptane, $\mathrm{CCl}_{4}$, and $n$-butyl ether, as shown in Table 1. In terms of the transition energy values, i.e., the $E_{\mathrm{T}}(30)$ values, the results are $31.1,31.1,32.4$, and $33.0 \mathrm{kcal} /$ mol, respectively, for the air/water interface, bulk $n$-heptane, bulk $\mathrm{CCl}_{4}$, and bulk $n$-butyl ether (Table 1 ). ${ }^{20}$ It is to be emphasized that compared with the very large shifts in transition energies relative to the bulk water value of $63.1 \mathrm{kcal} / \mathrm{mol}$, the $E_{\mathrm{T}}(30)$ values of the air/water interface and the bulk solvents heptane, $\mathrm{CCl}_{4}$, and dibutyl ether are very close to one other. This result also compares well with our previously reported $\mathrm{SH}$ measurement of the polarity of the air/water interface using DEPNA. ${ }^{9}$ The DEPNA study indicated that the polarity of the air/water interface $\left(\pi^{*}=0.22 \pm 0.07,76.9 \pm 0.6 \mathrm{kcal} / \mathrm{mol}\right)$ is similar to, but slightly less than, that of the bulk solvent carbon tetrachloride $\left(\mathrm{CCl}_{4}\right)$ on the $\pi^{*}$ scale $\left(\pi^{*}=0.26,76.2 \mathrm{kcal} / \mathrm{mol}\right)$ and likewise similar to bulk butyl ether $\left(\pi^{*}=0.21,76.9 \mathrm{kcal} /\right.$ mol). ${ }^{9}$ It is clear, therefore, that the polarity of the air/water interface determined using $E_{\mathrm{T}}(30)$ is similar to that determined using DEPNA. Both indicate that the air/water interface is nonpolar and is in the narrow polarity range corresponding to the bulk liquids carbon tetrachloride, butyl ether on the $E_{\mathrm{T}}(30)$ scale, and heptane on the $\pi^{*}$ scale (see Figure 4).

(b) DEPNA at Water/Organic Interfaces. The adsorption of DEPNA at water/organic interfaces is reduced compared with the absorption at the air/water interface. To obtain a detectable interface population, DEPNA concentrations several orders of magnitude greater ( $5 \mathrm{mM}$ in DCE and $20 \mathrm{mM}$ in $\mathrm{CB}$ ) than for experiments at the air/water interface were necessary. The apparently weak force driving DEPNA to the organic/water interface is consistent with the low interfacial tension of DCE/ water (32.8 dynes/cm) and $\mathrm{CB} /$ water $(37.4$ dynes $/ \mathrm{cm})$ interfaces compared with that of the air/water interface $(72.8$ dynes $/ \mathrm{cm})$.

The SH spectra of DEPNA $\left(I_{Z X X}^{(2) D E P N A}\right)$ at the DCE/water and $\mathrm{CB} /$ water interfaces are plotted in Figures 2 and 3, respectively. The CT band, due to a two-photon $\mathrm{SH}$ resonance, peaks at 415 $\pm 4 \mathrm{~nm}$ and $409 \pm 4 \mathrm{~nm}$ at the DCE/water and CB/water interfaces, respectively. The corresponding polarity values on the $\pi^{*}$ scale are listed in Table 1 (see Figure 4). The DCE/ water and $\mathrm{CB} /$ water interfaces have polarities that in bulk 
TABLE 1: $E_{\mathrm{T}}(30)$ and DEPNA Charge Transfer (CT) Band Peaks and Polarities for Various Solvents and Interfaces ${ }^{a}$

\begin{tabular}{|c|c|c|c|c|c|c|}
\hline & \multicolumn{3}{|c|}{$E_{\mathrm{T}}(30)$} & \multicolumn{3}{|c|}{ DEPNA } \\
\hline & $\begin{array}{c}\text { CT peak (nm) } \\
\text { expt (calcd) }\end{array}$ & $\begin{array}{c}E_{\mathrm{T}}^{\mathrm{N}} \text { scale } \\
\text { expt }(\text { calcd })\end{array}$ & $\begin{array}{c}E_{\mathrm{T}}(\mathrm{kcal} / \mathrm{mol}) \\
\operatorname{expt}(\mathrm{calcd})\end{array}$ & $\begin{array}{l}\text { CT peak }(\mathrm{nm}) \\
\text { expt }(\text { calcd })\end{array}$ & $\begin{array}{c}\pi^{*} \text { scale } \\
\text { expt (calcd) }\end{array}$ & $\begin{array}{c}E_{\pi}(\mathrm{kcal} / \mathrm{mol}) \\
\operatorname{expt}(\mathrm{calcd})\end{array}$ \\
\hline water & 453 & 1.00 & 63.1 & 429 & 1.32 & 66.4 \\
\hline air(vacuum) & (far-IR) & $(-0.93 \pm 0.02)$ & $(0)$ & 329 & -0.90 & 86.9 \\
\hline$n$-butyl ether & 866 & 0.07 & 33.0 & 372 & 0.21 & 76.9 \\
\hline $\mathrm{CCl}_{4}$ & 882 & 0.05 & 32.4 & 375 & 0.26 & 76.2 \\
\hline DCE & & & & 398 & 0.75 & 71.8 \\
\hline chlorobenzene $(\mathrm{CB})$ & & & & 394 & 0.67 & 79.6 \\
\hline$n$-heptane & 922 & 0.01 & 31.1 & 359 & -0.11 & 79.6 \\
\hline air/water & $920 \pm 20$ & $0.01 \pm 0.02$ & $31.1 \pm 0.7$ & $372 \pm 3(373)$ & $0.22 \pm 0.07(0.21)$ & $76.9 \pm 0.6(76.6)$ \\
\hline $\mathrm{CB} /$ water & & & & $409 \pm 4(411)$ & $0.96 \pm 0.07(1.00)$ & $68.9 \pm 0.7(69.1)$ \\
\hline DCE/water & & & & $415 \pm 4(414)$ & $1.08 \pm 0.07(1.04)$ & $68.9 \pm 0.7(69.1)$ \\
\hline
\end{tabular}

$0.52^{b}(0.51) \quad 47.7^{b}(47.1)$

${ }^{a}$ Values in brackets are calculated from eq 4 for the appropriate polarity scale. ${ }^{b}$ From ref 16.

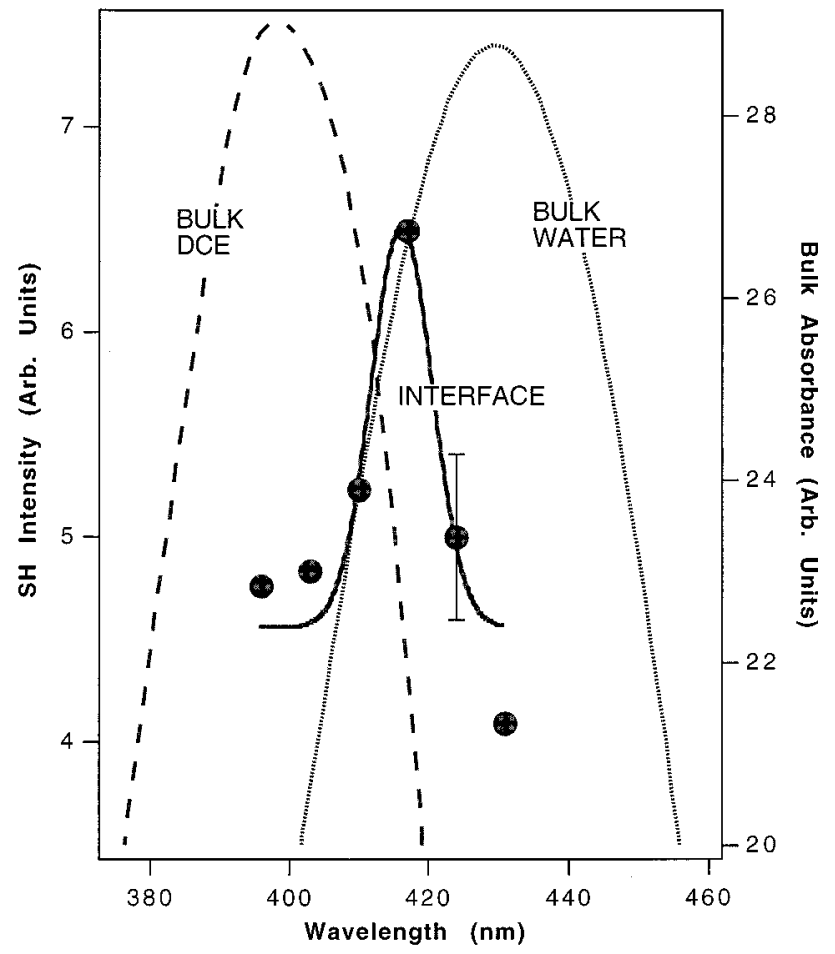

Figure 2. SH spectrum of DEPNA at the 1,2-dichloroethane (DCE)/ water interface. The circles are SH data for the ZXX element. The solid line is fit to eq 4 , yielding a peak position of $415 \pm 4 \mathrm{~nm}$. The dotted and dashed lines represent the absorption spectra in bulk water and DCE, respectively.

solvents correspond to ethylene glycol and benzyl alcohol, respectively.

(c) Generalized Polarity Scale. The results of the experiments with DEPNA at the air/water interface, the DCE/water interface, and the $\mathrm{CB} /$ water interface suggest a simple relationship between the interfacial polarity $\left(P_{\mathrm{A} / \mathrm{B}}\right)$ and the polarity of the constituent bulk phases $\left(P_{\mathrm{A}}\right.$ and $\left.P_{\mathrm{B}}\right)$ :

$$
P_{\mathrm{A} / \mathrm{B}}=\frac{P_{\mathrm{A}}+P_{\mathrm{B}}}{2}
$$

The validity of this relationship for the $\pi^{*}$ scale is supported by the good agreement between the experimentally determined polarity values using DEPNA and those predicted on the basis of the known $\pi^{*}$ scale polarity values of the bulk solvents. These results are summarized in Table 1 .

These results indicate that the solvation interactions of the interface molecules with the solvent molecules, which determine

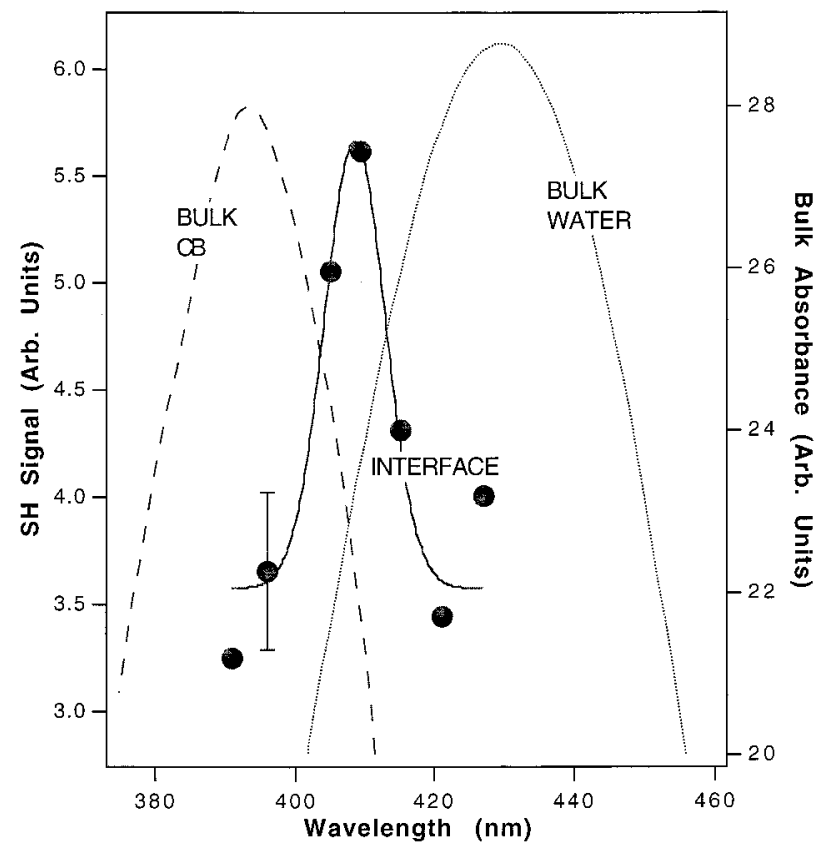

Figure 3. SH spectrum of DEPNA at the chlorobenzene (CB)/water interface. The circles are SH data for the ZXX element. The solid line is fit to eq 4, yielding a peak position of $409 \pm 4 \mathrm{~nm}$. The dotted and dashed lines represent the absorption spectra in bulk water and $\mathrm{CB}$, respectively.

the energy difference between the ground and lowest excited electronic states, are essentially the same for solute molecules whether located in the interface or in the bulk region of the solvent. This may be surprising because the local interfacial interactions of the adsorbate molecules with neighboring interfacial solvent molecules would be expected to differ from the solute-solvent interactions in the bulk region of the solvent. The asymmetry in forces imposed by the interface affects the absolute and mutual orientation of both the solute and solvent interfacial molecules and their mutual interactions. The arrangement of solvent molecules about the interfacial solute molecule is determined not only by the interfacial solutesolvent and interfacial solvent-solvent interactions but also by the interactions of both the interface solute and solvent molecules with the solvent molecules or vapor molecules, or solid that constitute the other bulk medium (vapor, liquid, solid) at the interface. Furthermore, a polar molecule will align itself such that its more polar part projects into the more polar solvent, e.g., water, and the less polar part into the less polar solvent. It would be unexpected if the local interfacial solvation interactions would be simply related to the individual bulk solvation when 


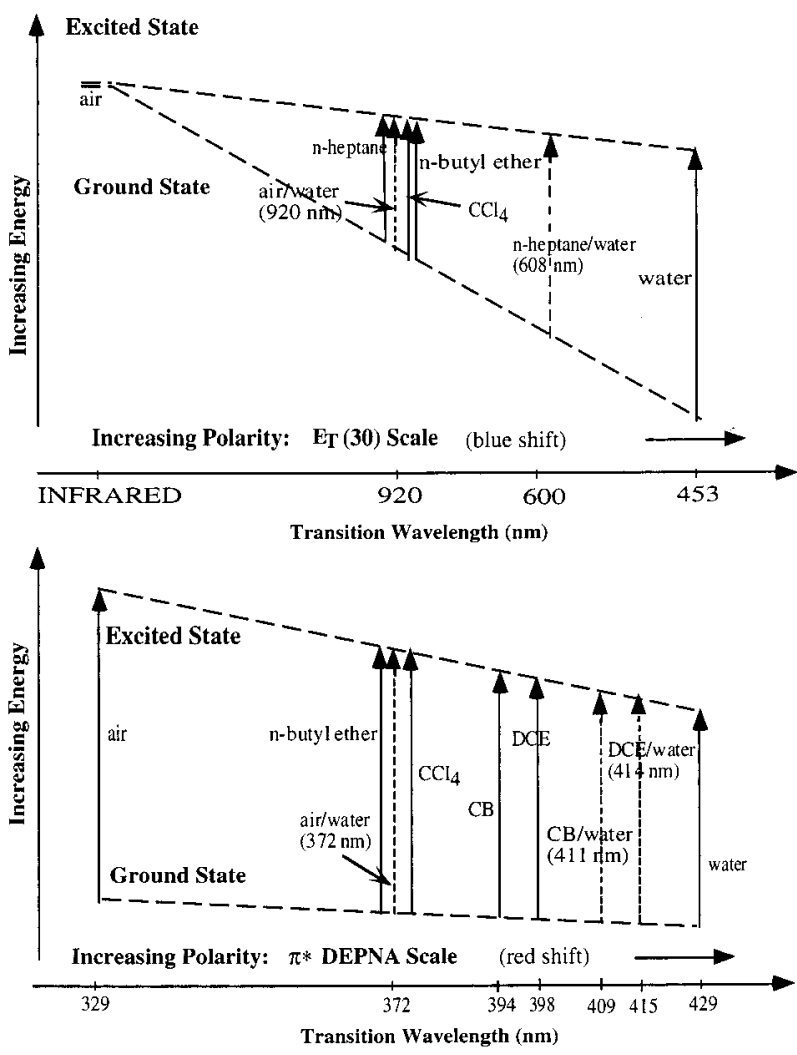

Figure 4. $E_{\mathrm{T}}(30)$ and $\pi$ polarity scales. Observed transition wavelengths for $E_{\mathrm{T}}(30)$ and DEPNA in bulk liquids and interfaces. CB is chlorobenzene and DCE is 1,2-dichloroethane. Solid arrows represent transitions in bulk liquids, and dotted arrows represent transitions at interfaces. The bracketed wavelengths are the predicted interface transition wavelengths.

different parts of the solute are projecting into different phases. An interpretation that is in accord with the interfacial polarity being directly related to the two bulk solvent polarities is a model in which the difference in excited- and ground-state solvation energies are determined chiefly by the long-range interactions of the interface solute molecules with the bulk solvent molecules. The polarity-sensitive solute molecules undergo large changes in their charge distributions, e.g., dipole moments, on photoexcitation. Dipole interactions are long-range interactions, and the larger number of bulk solvent molecules beyond the interface solvent shell will make their contribution the dominant one relative to the solvation energies. The finding that the interfacial polarity or, equivalently, that the differential solvation energies are equal to one-half of the arithmetic sum of the bulk polarities is a consequence of the fact that each solvent occupies only one-half of the total space and thus only contributes to one-half of the observed polarity. The fact that we can independently add the two bulk contributions to the polarity for a series of different interfaces, air/water, 1,2dichloroethane/water, and dichlorobenzene/water, brings into question a model in which the details of the different first-shell interfacial structures play the dominant role in determining the electronic transition energies. Nonetheless, it must be noted that only four different interfaces have been investigated up to this time and furthermore the possibility that the first shell dominates in some appropriate physical arrangement of the solvent about the interfacial solute molecule has not so far been decisively eliminated by the present studies, which have been restricted to water interfaces.

It would be of great practical and fundamental interest to determine if the relationship expressed in eq 5 holds in general for all interfaces and is independent of the polarity indicator molecule employed. To this end, we turn to the results of recent investigations of $E_{\mathrm{T}}(30)$ at alkane/water interfaces that use the method of total internal reflection. ${ }^{16}$ The large solvatochromic shift of $E_{\mathrm{T}}(30)$, its surface activity, and the fact that the polarity of the interface is so different from that of the constituent bulk phases ensure that the spectrum of interfacial species can easily be distinguished from those in the bulk. The CT band of $E_{\mathrm{T}^{-}}$ (30) was measured at the $n$-heptane/water, $n$-decane/water, and cyclohexane/water interfaces and found to peak at $600 \pm 10$ $\mathrm{nm}$ in each case. This corresponds to $E_{\mathrm{T}}^{\mathrm{N}}=0.52 \pm 0.03$. The prediction, based on eq 5 , yields an $E_{\mathrm{T}}^{\mathrm{N}}$ value of 0.51 , which is once again in excellent agreement with the experimental result. On the basis of these several results for DEPNA and $E_{\mathrm{T}}^{\mathrm{N}}$, it appears that eq 5 describes a general property of liquid interfaces.

There is no experimental measure of the position of the CT peak of $E_{\mathrm{T}}(30)$ in the gas phase. This value is important, since the gas phase (vacuum) CT peak serves as the zero solvation standard. The measurement of the CT band position of $E_{\mathrm{T}}(30)$ at the air/water interface and the relationship between interface and bulk polarities (eq 5) enables the band position in the gas phase to be determined. This relationship yields $E_{\mathrm{T}}^{\mathrm{N}}=-0.98$ \pm 0.03 , or $E_{\mathrm{T}}(30)=-1.05 \mathrm{kcal} / \mathrm{mol}$ for the gas phase. This corresponds to an approximate energy degeneracy for the ground state and excited states. To explore the reasonableness of this result, the energy difference between the ground state and excited configurations of $E_{\mathrm{T}}(30)$ in the gas phase was evaluated by semiempirical calculations using Professor Clark Still's Macromodel MM2 force field parameters. This calculation gives the energy difference as $12.4 \mathrm{kcal} / \mathrm{mol}$, corresponding to an electronic transition at $2300 \pm 10 \mathrm{~nm}\left(E_{\mathrm{T}}^{\mathrm{N}}=-0.56 \pm\right.$ $0.01)$. As with any calculation performed with a variational method, Macromodel yields an energy that is always greater than the actual energy. ${ }^{23}$ Therefore, with the same basis set, the energy of the excited configuration is not as well minimized as that of the ground state. A more accurate quantum mechanics calculation should give a smaller energy difference between these two configurations, i.e., a smaller gas-phase $E_{\mathrm{T}}(30)$ value and a transition at longer wavelength. As an extreme case, this gap could be so small that one could consider that these two electronic states have the same energy. The essentially degenerate ground and first excited states would make the electronic transition disappear, yielding $E_{\mathrm{T}}^{\mathrm{N}}=-0.93 \pm 0.02$, which is consistent with the value obtained from the $\mathrm{SH}$ measurement and the prediction based on eq 5 .

\section{Conclusions}

The SH spectroscopy of polarity indicator molecules at liquid interfaces has been used to investigate the polarity of the air/ water, 1,2-dichloroethane/water, and chlorobenzene/water interfaces. The consistency of the results obtained for the air/ water interface using two distinct indicator molecules, DEPNA and $E_{\mathrm{T}}(30)$, indicates that the $\mathrm{SH}$ method is useful as a method to probe interface polarity and that the air/water interface has a polarity corresponding to the narrow range of the bulk liquids butyl ether, $n$-heptane, and carbon tetrachloride. Of particular interest is the finding that the $\mathrm{SH}$ results lead to a simple relationship enabling prediction of interface polarities using known bulk polarities; the polarity of the interface between two fluids is the arithmetic average of the polarities of the constituent bulk phases. This simple relationship of interface polarity with the polarities of the bulk phases suggests that the difference in excited- and ground-state interface solvation energies of the 
adsorbed solute molecules could be chiefly due to long-range interface solute-bulk solvent interactions and not to local (first shell) solute-solvent interface interactions. Studies of interfaces other than the water interface of the present investigation are necessary to clarify these issues.

Acknowledgment. The authors thank the Division of Chemical Science of the Department of Energy for its support and the National Science Foundation for equipment support. Access to Professor Clark Still's Macromodel program at Columbia University is gratefully acknowledged. Thanks also go to Dr. Ruo Xu and Shisun Lei for help in the synthesis of DEPNA.

\section{References and Notes}

(1) Reichardt, C. Solvent and Solvent Effects in Organic Chemistry, 2nd ed.; VCH Publishers: Weinheim, 1988.

(2) Kamlet, M. J.; Abboud, J. L.; Taft, R. W. Prog. Phys. Org. Chem. 1981, 13, 485.

(3) Buncel, E.; Rajagopal, S. Acc. Chem. Res. 1990, 23, 226

(4) Marcus, Y. Chem. Soc. Rev. 1993, 409.

(5) (a) Hicks, J. M.; Vandersall, M. T.; Sitzmann, E. V.; Eisenthal, K B. Chem. Phys. Lett. 1987, 135, 413. (b) Langan, J. G.; Sitzmann, E. V.; Eisenthal, K. B. Chem. Phys. Lett. 1984, 110, 521-527.

(6) Fonseca, T.; Kim, H. J.; Hynes, J. T. J. Photochem. Photobiol., A 1994, $82,67-79$.
(7) Eisenthal, K. B. Acct. Chem. Res. 1993, 26, 636.

(8) Corn, R. M.; Higgins, D. A. Chem. Rev. 1994, 94, 107.

(9) Wang, H.; Borguet, E.; Eisenthal, K. B. J. Phys. Chem. B 1997, $101,713$.

(10) Lay, M. B.; Drummond, C. J.; Thistlethwaite, P. J.; Grieser, F. J. Colloid Interface Sci. 1989, 128, 602.

(11) Zachariasse, K. A.; Phuc, N. v.; Kozankiewicz, B. J. Phys. Chem. 1981, 85, 2676.

(12) Drummond, C. J.; Grieser, F.; Healy, T. W. Faraday Discuss. Chem. Soc. 1986, 81, 95.

(13) Warr, G. G.; Evans, D. F. Langmuir 1988, 4, 217.

(14) Michels, J. J.; Dorsey, J. G. Langmuir 1990, 6, 414.

(15) Lindley, S. M.; Flowers, G. C.; Leffler, J. E. J. Org. Chem. 1985 , 50,607 .

(16) Perera, J. M.; Stevens, G. W.; Grieser, F. Colloids Surf. A 1995, $95,185$.

(17) Shen, Y. R. Annu. Rev. Phys. Chem. 1989, 40, 327.

(18) Richmond, G. L.; Robinson, J. M.; Shannon, V. L. Prog. Surf. Sci. 1988, 28,1 .

(19) Kamlet, M. J.; Hall, T. N.; Boykin, J.; Taft, R. W. J. Org. Chem. 1979, 44, 2599 .

(20) Reichardt, C. Chem. Rev. 1994, 94, 2319.

(21) Behr, L. C.; Kirry, J. E.; MacDonald, R. N.; Todd, C. W. J. Am. Chem. Soc. 1946, 68, 1296.

(22) Yam, R.; Berkovic, G. Langmuir 1993, 9, 2109.

(23) Levine, I. N. Quantum Chemistry; Prentice Hall: New York, 1991.

(24) Kamlet, M. J.; Abboud, J. L.; Taft, R. W. J. Am. Chem. Soc. 1977, 99, 6027.

(25) Abboud, J. L.; Kamlet, M. J.; Taft, R. W. J. Am. Chem. Soc. 1977 99,8325 . 